\title{
Emotional dysfunction associated with diabetes in Mexican adolescents and young adults with type-1 diabetes
}

\author{
Israel Lerman-Garber, MD, ${ }^{(1)}$ C onsuelo Barrón-U ribe, MD, (2) Raúl Calzada-León, MD, (3) \\ Moisés Mercado-A tri MD, ${ }_{1}^{(4)}$ Rafael Vidal-Tamayo, MD, ${ }^{(1)}$ Silvia Q uintana, MD, ${ }^{(3)}$ María Elena Hernández, BS, ${ }^{(2)}$ \\ María de la Luz Ruiz-Reyes, BS, ${ }^{(3)}$ Laura Elena Tamez-Gutiérrez, MD, ${ }^{(1)}$ \\ Elisa $\mathrm{N}$ ishimura-Meguro, MD, ${ }^{(2)}$ Antonio R Villa, MD. ${ }^{(5)}$
}

\begin{abstract}
Lerman-Garber I, Barrón-Uribe C, Calzada-León R, Mercado-Atri M,Vidal-Tamayo R, Quintana S, Hernández ME, Ruiz-Reyes ML,Tamez-Gutiérrez LE, Nishimura-Meguro E, VillaAR. Emotional dysfunction associated with diabetes in Mexican adolescents and young adults with type-1 diabetes. Salud Publica Mex 2003;45:13-18. The English version of this paper is available too at: http://www.insp.mx/salud/index.html
\end{abstract}

\begin{abstract}
A bstract
Objective. To assess the emotional dysfunction asso ciated with diabetes in Mexican young individuals with type-1 diabetes. Material and Methods. A cross-sectional survey was conducted to perform a complete clinical and psychosocial evaluation of 93 consecutive type-1 diabetes patients, aged 14 to 30 years. Results. Adolescents had higher scores of emotional dysfunction related to diabetes and a diminished knowledge in diabetes-related areas.A multivariate logistic regression model showed that an inadequate emotional response to diabetes (high problem areas in diabetes or PAID scores) was mainly associated with a poor glycemic control $(0 \mathrm{R}=2.9,95 \% \mathrm{Cl}$ 0.9-9.7, $\mathrm{p}=0.09)$. Apprehension about the future and the possibility of serious complications had the highest mean PAID score in all age groups. Conclusions. N ew strategies should be develo ped to improve the routine care and support of young individuals with type-1 diabetes.The English version of this paper is available too at: http://www.insp.mx/salud/index.html
\end{abstract}

Key words: type-1 diabetes; emotional dysfunction; glycemic control; Mexico

\author{
Lerman-Garber I, Barrón-U ribe C, Calzada-León R, \\ Mercado-Atri M,Vidal-Tamayo R, Quintana S, \\ Hernández ME, Ruiz-Reyes ML,Tamez-Gutiérrez LE, \\ Nishimura-Meguro E, VillaAR. \\ Disfunción emocional asociada a diabetes en adolescentes \\ y adultos jóvenes mexicanos con diabetes tipo 1. \\ Salud Publica Mex 2003;45:13-18. \\ El texto completo en inglés de este artículo también \\ está disponible en: http://www.insp.mx/salud/index.html
}

\section{Resumen}

Objetivo. Investigar la disfunción emocional asociada con la diabetes en jóvenes mexicanos con diabetes tipo 1. Material y métodos. Estudio transversal que incluyó la evaluación clínica y psicosocial de 93 pacientes con diabetes tipo 1 con edades comprendidas entre los 14 y los 30 años. Resultados. LOS adolescentes como grupo presentaron mayor disfunción emocional aso ciada con la diabetes y menores conocimientos en las diferentes áreas de diabetes. En un análisis de regresión logística multivariado, una respuesta emocional inadecuada (calificación alta de áreas problema en diabetes o PAID, por sus siglas en inglés), se asoció principalmente con un mal control glucémico $(0 \mathrm{R}=2.9 ; 95 \%$ Cl 0.9-9.7 $p=0.09$ ). La preocupación acerca del futuro y la posibilidad de desarrollar complicaciones se asoció con las calificaciones más altas de PAID en todos los grupos de edad. Conclusiones. Es una imperiosa necesidad desarrollar nuevas estrategias que permitan mejorar el cuidado y brindar más apoyo a los jóvenes con diabetes tipo 1. El texto completo en inglés de este artículo también está disponible en: http://www.insp.mx/salud/index.html

Palabras clave: diabetes tipo 1; disfunción emocional; control glucémico; México

The present study was previously presented as a poster at the $61^{\text {st }}$ Scientific Session of the American Diabetes Association.

(1) Departamento de Endocrinología, Instituto N acional de Ciencias Médicas y Nutrición Salvador Zubirán. México D.F., México

(2) Departamento de Endocrinología, Centro Médico N acional Siglo XXI, Instituto Mexicano del Seguro Social (IMSS), México, D.F., México.

(3) Servicio de Endocrinología Pediátrica, Instituto Nacional de Pediatría, México, D.F., México.

(4) Departamento de Endocrinología Pediátrica, Centro Médico N acional Siglo XXI, IMSS, México, D.F., México.

(5) Unidad de Epidemiología Clínica, Instituto N acional de Ciencias Médicas y N utrición Salvador Zubirán, México, D.F., México.

Received on: A ugust 21,2001 • Accepted on: 0 ctober 15,2002

Address reprint requests to: Israel Lerman MD. D epartamento de Endocrinología y Metabolismo. Instituto N acional de Ciencias Médicas y N utrición Salvador Zubirán.Vasco de Q uiroga 15.Tlalpan, 14080 México, D.F., México.

E-Mail: lerman@ netservice.com.mx 
$\mathrm{E}$ arly adulthood is the developmental period during which individuals assume a greater degree of independent functioning and take on adult roles. A chronic illness like diabetes could hinder the development of these aspects of the life cycle.

Many studies have demonstrated that psychosocial stress during adolescence is often associated with neglect to follow treatment guidelines, as well as with a higher risk of poor metabolic control requiring hospitalization and, eventually, with the development of long-term complications. ${ }^{1-9}$

In young adults with type- 1 diabetes, the primary concern is the actual or potential manifestation of physical damage related to chronic disease. Some patients are overwhelmed by the fear of physical impairment, whereas others actively suppress these concerns. $^{2-4,10-16}$ The psychological demand of leaving home and learning to live on one's own is quite a challenge. The need to find a new physician adds to these problems. However, early adulthood could be the end of a stormy period, and many patients return for clinical advice and guidance. After years of poor glycemic control, many patients begin to take life and their illness more seriously, and intensive insulin treatment programs can succeed.

Mexico has a population currently estimated at 100 million inhabitants. Type- 1 diabetes is uncommon (the estimated incidence is $4 / 100000){ }_{1}^{17}$ but has a high rate of complications at early stages of the disease. In Mexico's partially socialized Health Care System, most of the children and adolescents with type- 1 diabetes are expected to be treated in Pediatric Endocrine Centers, and at age 17-18 they are transferred to Internal Medicine services. The aim of the present study was to investigate the emotional dysfunction associated with diabetes in Mexican adolescents and young adults with type- 1 diabetes.

\section{Material and Methods}

The present study is a cross-sectional survey. The sample population consisted of type- 1 diabetes patients aged 14 to 30 years, diagnosed with diabetes as children or adolescents. Patients diagnosed within the year, or those recently admitted (within the previous month) for in-patient treatment, were excluded.

Between March and September 2000, 93 consecutive type- 1 diabetes patients were included in the study. Participants were selected during their routine outpatient visits in different locations (Departments of Endocrinology of the Instituto Nacional de Ciencias Médicas y Nutrición Salvador Zubirán, Instituto Nacional de Pediatría, Centro Médico Siglo XXI, and pri- vate clinics). Clinical, psychosocial and demographic data were obtained. The patients answered two established self-report, previously validated questionnaires: one related to their knowledge about different diabetes issues ${ }^{18}$ and the other was the Problem areas in diabetes (PAID) questionnaire. PAID scores are converted to a 0-100 scale, with higher scores indicating greater emotional distress. ${ }^{19-21}$ In addition, young adults over 18 years of age answered specific questions and described the transitional period of diabetes care between adolescence and young adulthood. Poor glycemic control was defined as a hemoglobin A1c (HbA1c) level $>9 \%$ (non-diabetic range $<6.1 \%$ ).

Analyses of previously classified variables was performed to assess associations. The sociodemographic and clinical variables were compared for the different age groups (adolescents were defined as the 14-17 year-old group). A chi-squared test was used to test differences between categorical variables. For continuous variables, the non-parametric KruskalWallis test was employed. Odds ratios were obtained by logistic regression analysis. A 3-point Likert scale (worse, better, or no change) was used to evaluate how young adults perceived changes in their diabetes care since their adolescent years). Questions were related to: glycemic control, meal planning, exercise, home blood glucose (BG) monitoring, adherence to insulin regimens, concern about diabetes, episodes of severe hypoglycemia and/or diabetic ketoacidosis (DKA).

The PAID questionnaire was previously translated, back-translated, and validated in the sample population. Its psychometric properties were tested in a pilot group of 20 Mexican diabetes patients, with a Cronbach's alpha coefficient of reliability for each item $>0.90 .{ }^{21}$ In the present study, the internal consistency of the entire questionnaire had a Cronbach's alpha reliability coefficient of 0.945 . The diabetes knowledge scale involved a similar process and previous validation in our population. ${ }^{18}$ Results were expressed in numerical values. Data are presented as mean \pm SD where appropriate. The study protocol was approved by the Medical Ethics Committee.

\section{Results}

The clinical and sociodemographic data of the studied population is shown in Table I. Significant differences were observed in the different age groups. Adolescents as a group had higher scores in emotional dysfunction related to diabetes (PAID) and diminished knowledge about diabetes. Adolescents had poorer glycemic control, although it did not reach statistical significance. The 25-30 age group tended to have better glycemic 
Table I

\section{Clinical and sociodemographic characteristics by age group. MeXico City, 2000}

\begin{tabular}{|c|c|c|c|c|}
\hline Age group & 14-17 & $18-24$ & $>24$ & $p$ value \\
\hline$n$ & 21 & 55 & 17 & \\
\hline $\operatorname{Sex}(M / F)$ & $12 / 9$ & $31 / 24$ & $5 / 12$ & 0.11 \\
\hline Very poor income class (\%) & 38 & 33 & 24 & 0.14 \\
\hline Moderate alcohol intake (\%) & 10 & 26 & 29 & 0.13 \\
\hline Smoking (\%) & 5 & 26 & 24 & 0.13 \\
\hline Eating disorders (\%) & 10 & 6 & 5 & 0.63 \\
\hline Years since diagnosis (mean \pm SD) & $6+-4$ & $9+-4$ & $15+-3$ & 0.001 \\
\hline Poor glycemic control (\%)* & 40 & 32 & 18 & 0.21 \\
\hline No BG monitoring (\%) & 14.3 & 43.6 & 47.1 & 0.01 \\
\hline Intensive insulin program (\%) & 43 & 31 & 29 & 0.27 \\
\hline Severe hypoglycemia that year (\%) & 10 & 16 & 18 & 0.47 \\
\hline Episode of DKA that year (\%) & 10 & 16 & 6 & 0.82 \\
\hline Proliferative retinopathy (\%) & 5 & 16 & 29 & 0.04 \\
\hline Peripheral neuropathy (\%) & 0 & 7.3 & 23.5 & 0.01 \\
\hline Clinical proteinuria (\%) & 9.5 & 9.1 & 41.2 & 0.01 \\
\hline End-stage renal disease (\%) & 0 & 2 & 12 & 0.05 \\
\hline Autonomic neuropathy (\%) & 0 & 9.1 & 17.6 & 0.05 \\
\hline High PAID score (\%) $)^{\ddagger}$ & 67 & 53 & 24 & 0.01 \\
\hline High diabetes knowledge scores $(\%)^{\S}$ & 57 & 56 & 94 & 0.05 \\
\hline \multicolumn{5}{|c|}{ 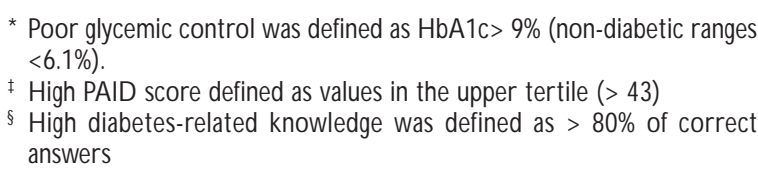 } \\
\hline
\end{tabular}

control and lower PAID scores, but fewer individuals were doing BG monitoring or intensive insulin treatment programs, and the prevalence of diabetes-related complications was high. In a multivariate model of the logistic regression analysis, inadequate emotional response to diabetes (high PAID scores) was mainly associated with poor glycemic control (OR= 2.9; $95 \%$ CI 0.9-9.7, $p=0.09$ ). When metabolic control was included in the model, two other variables had an inversely significant association; higher educational levels $(\mathrm{OR}=0.09 ; 95 \% \mathrm{CI} 0.01-0.8, p=0.03)$ and evidence of an episode of severe hypoglycemia within the year (OR= 0.3; 95\% CI 0.07-1.1, $p=0.06$ ). Age, sex, income, activity, marital status, treatment regimen, alcohol intake, diabetes duration, home BG monitoring or history of DKA did not show a significant association. Apprehension about the future and the possibility of serious complications had the highest mean PAID score in all age groups. Adolescents had significantly higher PAID scores in the following items: feeling scar- ed about living with diabetes; feeling guilty about not complying with diabetes treatment, and dissatisfaction with the physician. There was a trend about feeling more discouraged with the diabetes regimen and problems with interactions related to diabetes with family/ friends (Table II).

Specific questions answered by patients 18 years and older, showed that young adulthood was perceived as a moment in life with increased concerns about glycemic control and the development of late complications of the disease. There was a trend for diminished DKA episodes and more episodes of hypoglycemia. Young adults described how they experienced the transition period from adolescence to young adulthood. Some of their main comments were the following: "I am more worried now but I just can't stay under control; I had a very negative and pessimistic perspective"; "As an adolescent I was "untouchable"; "I couldn't accept my illness"; "I was very rebellious"; "I did not take my illness seriously"; "I was always frustrated"; "I used to feel sad and inferior to others"; "I have learned to live with diabetes"; "I am more responsible"; "I have stopped letting diabetes run my life"; "I am recovering happiness after living many years as if I where in a hole (antisocial and frightened)"; "It changes from a sickness to a life-style"; "My control has nothing to do with my age, it depends on other circumstances like problems at home, school or work"; "I felt my mother on my back all the time"; "Before I was not alone, I had my family's support"; "Since my parents have not been paying much attention, I have not taken care of my health"; "I knew that even if I didn't pay attention to the doctors or my parents, nothing was going to happen to me"; "I lied and was bothered by the doctors, who made me feel guilty about my HbA1c levels"; "My new doctor examined me and asked me about my diabetes, but he is not really interested in me"; "It helped me a lot to meet other young people with diabetes"; "Now I am worried about complications and my future"; "I was not in control until I became pregnant"; "Now with so many activities and responsibilities, its more difficult to care for my health"; "Now I realize that I could have prevented the complications".

\section{Discussion}

The present study was done with the main objective to describe the emotional dysfunction associated with diabetes in Mexican adolescents and young adults with type-1 diabetes. Adolescents as a group had higher scores in emotional dysfunction related to diabetes (PAID), and diminished knowledge in diabe- 
Table II

PAID mean scores for the different Age groups. Mexico City, 2000

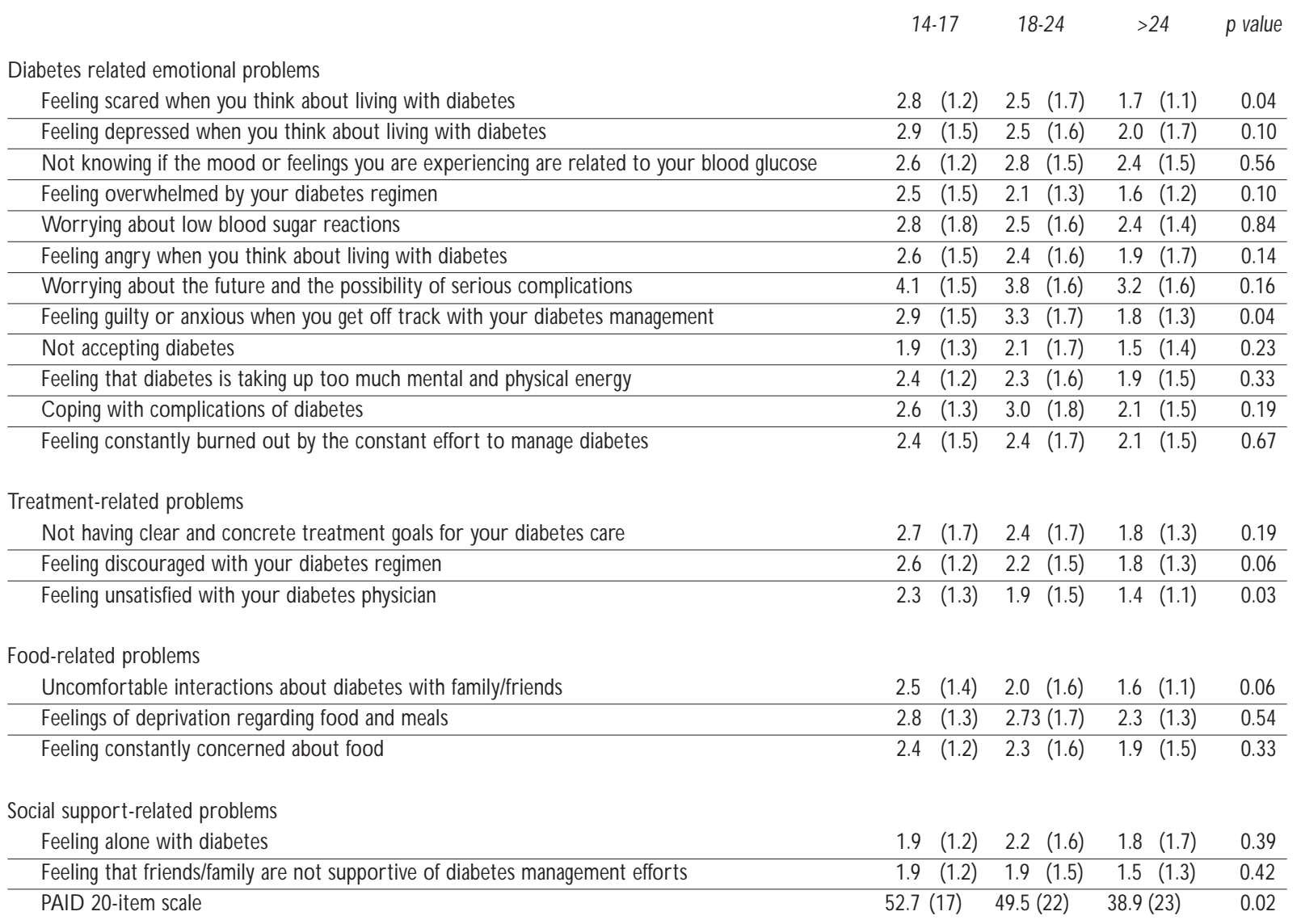

tes-related areas. The 25-30 age group, which was composed mostly by women, showed a trend for a better glycemic control and lower PAID scores; however, fewer individuals were doing BG monitoring or had intensive insulin treatment programs. The improvement in metabolic control in this age group is likely related to a better adherence to the diabetes treatment program. It does not mean, however, that intensive programs do not result in a better glycemic control.

The Problem Areas in Diabetes Scale ${ }^{19-21}$ is a brief self-report measure of diabetes-related distress that has been found to be valid and clinically useful in type-1 and type- 2 diabetes patients. PAID scores have been found to show positive associations with $\mathrm{HbA1c}$, and are a major predictor of poor adherence to treatment not involving general emotional distress. In the present study, an inadequate emotional response to diabetes was mainly associated with poor glycemic control, and inversely, with higher educational levels and recent evidence of severe hypoglycemia. Adolescents had higher scores in the following items; feeling scar- ed about living with diabetes; feeling guilty about not complying with treatment programs, and dissatisfaction with their attending physician. There was also a trend towards feeling more discouraged with the diabetes regimen and uncomfortable with interactions related to diabetes issues with family/friends.

In a study comparing type-I diabetic subjects in US and Dutch population, ${ }^{22}$ the mean PAID score was $33 \pm 22$ and $25 \pm 19$ respectively, compared with $48 \pm 21$ in the present study. The differences observed are only partially related to the youngest age in our studied population. As in the present study, lower $\mathrm{HbA1}$ c levels were associated with less emotional dysfunction related to diabetes, and a younger age was related to higher PAID scores.

Critical tasks of young adulthood include the development of a comfortable identity, the capacity to enjoy intimate relationships, the ability to preserve a previously developed sense of autonomy and productivity, and the acquisition of more mature defenses against adversity. Concerns about the presence of a 
chronic illness may inhibit this progression. Some young people make decisions based on incomplete information, which changes the course of their lives. For example, some avoid marriage or permanent relationships, or fail to get involved in long-term commitments. $^{9-11,14-16}$

Most of the medical literature includes information from patients who have had an adequate medical follow-up. In a previous report, ${ }^{23}$ we studied 209 type-1 diabetes patients who attended two of the main Pediatric Endocrine Clinics in Mexico City, and left those Centers because of age (as they became young adults). We could not obtain information or confirm a medical follow-up in $71 \%$ of them; eight were known to be dead (seven from diabetes-related complications). Presumably, most patients were seen by primary care physicians, or by no physicians at all. Only $62(29 \%)$ of the patients, could be reached by phone, telegram or during their routine clinical appointments. Reviewing their old clinical records as adolescents, two thirds had poorly controlled diabetes and $16 \%$ had proliferative retinopathy. This information leads us to reflect upon the true story of diabetes, particularly in underdeveloped countries or in very low income groups.

Why are so many patients lost to follow-up?, and What happens to them?, are two important questions that deserve an answer, and are likely related to a diversity of factors: The scarce economic and social resources compel patients to not leaving their work for a medical appointment (consequently, most of them do not even mention that they have diabetes for the fear of losing their job). Others, as young adults, may lose the family health coverage offered by the Social Security System. There may be erroneous attitudes towards a disabled child (in this case with diabetes), more common in the poor income classes, that leave these patients with no other alternative but to deny their illness. Patient attitudes such as reluctance to see another physician, take on more responsibilities, be overwhelmed by other problems, and the belief that they will be safe just by following the usual indications, are some of the coping strategies used by patients. Unfortunately, patients usually return too late to be followed and treated for their diabetes, when the majority of them have already developed serious complications of the disease. The latter is usually the main reason why they return for medical follow-up.

Only a few studies have examined the impact of diabetes in patients as they experience the transition into young adulthood. Lloyd et all ${ }^{10}$ observed that type1 diabetes patients where more socially isolated than healthy controls. Tebbi et al ${ }^{11}$ found that general wellbeing was lower in diabetes patients, but that they were able to adjust well in terms of employment-related issues. Increased rates of depression and high, but significant rates of suicide have not been observed. ${ }^{12,13}$ Other authors have suggested that there may be an increased prevalence of eating or other psychiatric disorders. ${ }^{24}$ As it has become obvious, diabetes in someone with immature psychosocial defenses is more likely to require increased structure and support for treatment. ${ }^{1-3,15}$

Other studies suggest that type- 1 diabetic young patients do not experience lower self esteem, more symptomatic distress, or personality disorders. ${ }^{14-16}$ Adolescents were found to be carrying out the developmentally appropriate tasks involved in the transition to adulthood. However, they perceived a lower competence in social relationships, but diabetes did not have a clear negative impact on their adjustment as young adults. These findings were observed in a Caucasian population reasonably well educated and with few individuals from lower socioeconomic strata. It is likely that type- 1 diabetes causes more serious psychosocial problems in patients from minority populations, who are often less educated and from a lower socioeconomic class. ${ }^{14}$

In the present study, young adulthood was associated with a better metabolic control in females (those reached by the survey and who had a medical follow-up). This could be related to the fact, that they became more concerned about the near future (marriage, motherhood, complications).

Adolescence and early adulthood is different in Mexico than in most developed countries. The family bounds are closer, young adults must obtain their own economical support at early ages and independence from the family occurs only several years later.

In a recent study comparing health care indicators in type- 1 diabetes patients, it was concluded that other components of the health care structure (including free, and/or easy access to insulin pens and selfmonitoring of blood glucose supplies) may be critical. ${ }^{24}$ In Mexico, because of poverty and scarce medical and family resources, intensive insulin treatment programs are a real option for less than $5 \%$ of our diabetic population. ${ }^{17}$

Diabetes regimens include many aspects that are difficult to comply with; it is a chronic disorder, lifestyle changes are required, treatment is complex, intrusive, and inconvenient, and prevention instead of symptom reduction or cure is usually the main goal. Variables that are known to affect adherence to treatment include the type and duration of diabetes, evidence of late complications, availability of socioeconomic resources, educational background, culture, and the individual's personality. ${ }^{1,25}$ 
As evidenced in this and other studies, most young adults have no clear concept of chronic illness and prevention, therefore, this is not a problem exclusive of this age group. It is a great challenge, particularly during the transition period from adolescence to early adulthood. It is necessary to discuss carefully the prognosis of the quality and duration of life and to review the known facts about the long-term outcome of diabetes. The relevance of relationship building, assessing expectations, realistic goal setting, and team and family support is unquestionable. Cost-effective approaches and optimization of resources must always be kept in mind.

In conclusion, the identification of psychological and behavioral problems in adolescents and young adults with diabetes is mandatory. There is a need to develop new strategies that may help to improve the follow-up and treatment of type-1 diabetic patients.

\section{References}

1. LaG reca AM, Follansbee D, Skyler JS. D evelopmental and behavioral aspects of diabetes management in youngsters. $\mathrm{C}$ hild $\mathrm{H}$ ealth $\mathrm{C}$ are 1990;19:132-139.

2. Tattersall RB, Lowe J. Diabetes in adolescence. Diabetologia 1981;20: 517-523.

3. The Diabetes Control and Complications Trial Research Group. The effect of intensive treatment of diabetes on the development and progression of long-term complications in insulin-dependent diabetes mellitus. N Engl J Med 1993;329:977-986.

4. Hanson CL, Harris MA, Relyea G, Cograng GA, Carle DL, Burghen GA. Coping styles in youths with insulin-dependent diabetes mellitus. J Consult Clin Psychol 1989;57:644-651.

5. Charron-Prochownik D,Arslaninan S.W omen with diabetes:The adolescent years: A case study and review. Diabetes Spectrum 1997;10: 180-184.

6. Delamater AM, Kurtz SM, Bubb J, W hite N H, Santiago JV. Stress and coping in relation to metabolic control in adolescents with type-1 diabetes. Dev Behav Pediatr 1987;8:136-140.

7. Golden MP. Incorporation of quality-of-life considerations into intensive diabetes management protocols in adolescents. Diabetes $C$ are 1998; 21:885-886.

8. Jacobson AM, Hauser ST, W ertlieb D, W ofsdorf Jl, 0 rleans J,Vieyra M. Psychological adjustment of children with recently diagnosed diabetes mellitus. Diabetes Care 1986:9:323-329.
9. Grey M, Boland EA, D avidson M,Yu C, Sullivan-Bolyai S,TamborlaneW V. Short-term effects of coping skills training as adjunct to intensive therapy in adolescents. Diabetes Care 1998:21:902-908.

10. Lloyd CE, Robinson N ,Andrews B, Elston MA, Fuller JH.A re the social relationships of young insulin-dependent diabetes patients affected by their condition? Diabet Med 1993;10:481-485.

11. Tebbi C, Bromberg C, Sills I, C ukierman J, Piedmonte M. Vocational adjustment and general well-being of young adults with ID D M. Diabetes Care 1993;13:983-989.

12. Mayou R, Peveler R, D avies B, Mann J, Fairburn C. Psychiatric morbidity in young adults with insulin-dependent diabetes mellitus. Psychol Med 1991; 21:639-645

13. Kyvik K, Satenager E, G reen A, Svendsen A. Suicides in men with ID D M . Diabetes Care 1994;17:210-213.

14. Jaco bson AM, Hauser ST, W illet JB, D vorak R, W olpert H, Herman L et al. Social relationships among young adults with insulin dependent diabetes mellitus: Ten year follow-up of an onset cohort. Diabet Med 1997; 14:73-79.

15. Jacobson AM, Hauser ST,W illwt JB, W olfsdorf JI, D vorak R, Herman L et al. Psychological adjustment to ID D M: 10-year follow-up of an onset cohort of child and adolescent patients. Diabetes C are 1997;20:811-818. 16. Dunn SM, Turtle JR. The myth of the diabetic personality. Diabetes Care 1981:4:640-646.

17. Lerman I, Q uibrera R, Gómez-Pérez FJ. Epidemiology of diabetes in Mexico. En: Ekoe JM, Zimmet P,W illiams R, ed.The epidemiology of diabetes: An international perspective. Londres: John W iley \& Sons, 2001: 177-186.

18. Bautista MS,A guilar-Salina CA, Lerman GI,Velasco ML, Castellanos R, Zenteno $E$ et al. Diabetes knowledge and its determinants in a Mexican Population.The Diabetes Educator 1999;25:3-9.

19. Polonsky W H,Anderson BJ, Lohrer PA,W elch G, Jacobson AM, A ponte JE et al. Assessment of diabetes-related distress. Diabetes Care 1995; 18:754-760.

20. Welch GW, Jacobson AM, Polonsky W H. The Problem Areas in Diabetes Scale: An evaluation of its clinical utility. Diabetes Care 1997;20: 760-766.

21. González-U resti X, LópezAJC,A guilar SC, G arcía G E, G ómez-Pérez FJ, Lerman GI. Disfunción emocional y diabetes. XIV Congreso Panamericano de Endocrinología; 1997;noviembre 21-25;C ancún, México: 29.

22. Snoek FJ, Pouwer F,W elch GW, Polonsky W H. Diabetes-related emotional distress in Dutch and US diabetes patients. Cross-cultural validity of the Problem Areas in Diabetes Scale. Diabetes Care 2000;23:13051309.

23. Lerman GI, Barrón C, C alzada LR, Mercado M,Vidal R, Q uintana S et al. Transition from adolescence to young adulthood in diabetes care (Abstract). Diabetes 2001;50(2):393A.

24. Rodiri GM, Daneman D. Eating disorders and IDDM:A problematic association. Diabetes Care 1992;15:1402-1411.

25. Tabak AG, Tamas G, Zgibor J, W ilson R, Becker D, Kerenyi Z et al. Targets and reality:A comparison of health care indicators in the US (Pittsburgh Epidemiology of D iabetes Complications Study) and Hungary (D iab Care Hungary). Diabetes Care 2000;23:1284-1289. 